\title{
Étude génétique de la représentation des femmes dans Un homme pareil aux autres de René Maran
}

Tina Harpin et Laura Gauthier Blasi

\section{OpenEdition}

\section{Journals}

Édition électronique

URL : https://journals.openedition.org/coma/7444

DOI : $10.4000 /$ coma.7444

ISSN : 2275-1742

Éditeur

Institut des textes \& manuscrits modernes (ITEM)

\section{Référence électronique}

Tina Harpin et Laura Gauthier Blasi, «Étude génétique de la représentation des femmes dans Un homme pareil aux autres de René Maran », Continents manuscrits [En ligne], 17 | 2021, mis en ligne le 15 octobre 2021, consulté le 09 janvier 2022. URL : http://journals.openedition.org/coma/7444 ; DOI : https://doi.org/10.4000/coma.7444

Ce document a été généré automatiquement le 9 janvier 2022.

\section{cc) $(\mathrm{S})$}

Continents manuscrits - Génétique des textes littéraires - Afrique, Caraîbe, dispora est mis à disposition selon les termes de la licence Creative Commons Attribution - Pas d'Utilisation Commerciale - Pas de Modification 4.0 International. 


\title{
Étude génétique de la représentation des femmes dans Un homme pareil aux autres de René Maran
}

\author{
Tina Harpin et Laura Gauthier Blasi
}

1 Avant sa version définitive de 1947 aux éditions Arc-en-ciel, rééditée par Albin Michel en 1962, le roman Un homme pareil aux autres ${ }^{1}$ est paru sous des titres différents et dans des versions plus ou moins longues. Il a été réécrit sur plusieurs décennies comme l'attestent divers documents imprimés et plusieurs manuscrits ${ }^{2}$. Ce texte "instable » dont le processus de création interroge encore, est l'un des romans les plus connus et les plus personnels ${ }^{3}$ de René Maran. Intellectuel noir au parcours brillant dans la France coloniale, né de parents guyanais en Martinique en 1897, administrateur colonial devenu écrivain, récompensé par le prix Goncourt en 1921 pour Batouala, et auteur d'une œuvre littéraire considérable ${ }^{4}$ à sa mort à Paris en 1960, Maran a commencé à rédiger Un homme pareil aux autres au début des années 1920 en l'intitulant «Le Roman d'un nègre $»^{5}$. Ce qui devait être pour lui d'abord un « livre du souvenir $»^{6}$ prit la forme d'un récit de voyage et d'un roman d'amour. Jean Veneuse, le personnage principal, fonctionnaire colonial noir en Afrique équatoriale française raconte, presque sur le mode de l'écriture diariste ${ }^{7}$, sa séparation puis ses retrouvailles avec Andrée Marielle, femme blanche éduquée, fille du poète Louis Marielle, qui vit à Paris et à qui il a osé déclarer sa flamme. Commencé pendant les Années folles, période où la transformation des mœurs célèbre l'amour et l'idée du mariage par amour ${ }^{8}$, ce récit aborde la question sensible des couples mixtes, notamment, du couple formé par l'homme noir et la femme blanche.

2 Le couple mixte n'est pas un sujet ignoré par les romans de l'époque qui évoquent surtout les amours plutôt coloniales d'hommes blancs et de femmes non blanches (asiatiques, noires, maghrébines...). Roger Little note que la femme blanche reste souvent en marge ${ }^{9}$, réduite à celle qui attend, en « métropole », le retour du héros parti 
en terres inconnues, affronter indigènes et bêtes sauvages comme pour assurer sa virilité $^{10}$ avant de se marier. Ce scénario se retrouve en partie dans Un homme pareil aux autres, puisqu'Andrée, l'élue de Veneuse fait figure de Pénélope. Mais le héros noir donne à l'intrigue une portée transgressive car Veneuse brise ce que Roger Little appelle le «tabou inhérent à la mentalité coloniale qui l'empêcherait [...] d'imaginer qu'une Blanche puisse tomber amoureuse d'un noir $\aleph^{11}$. À travers le récit à la première personne et le «je » de Veneuse ${ }^{12}$, René Maran donne à entendre la voix et la pensée de l'Autre racisé qu'est l'homme noir dans un contexte marqué par les préjugés et le racisme. Son héros explique au lecteur que «le livre que voici n'est, au fond, que le voyage d'une race à une autre race et d'un cœur à un autre cœur » (HPA, 13). En parlant du cœur, Veneuse dévoile son humanité et laisse entrevoir la complexité de son être. Les critiques l'ont bien compris, tel Buata Malela qui résume la question ainsi: "Veneuse est bien "un homme pareil aux autres", grâce notamment à l'amour qui sert de remède à la violence des Français des colonies ${ }^{13}$. » Ce sont précisément les limites et contradictions de l'écriture sentimentale de l'amour dans le roman que nous voulons étudier dans cet article, en analysant les portraits des femmes ${ }^{14}$ au fil des différentes versions du roman.

3 L'étude génétique d'Un homme pareil aux autres permet d'identifier les choix opérés par René Maran dans la mise en scène de son héros sentimental, si proche de sa persona d'auteur. Comme l'explique Lourdes Rubiales, le choix du nom de Jean Veneuse est une " négation du pacte autobiographique par l'hétéronymie » mais « n'a pas empêché pour autant le lecteur d'[...] établir des rapports d'identité entre le héros-narrateur et l'auteur de $c[\mathrm{e}]$ text $[\mathrm{e}] »^{15}$. Ainsi, outre la portée sociale indéniable que Maran conférait à son roman ${ }^{16}$, l'autre enjeu de ce texte d'inspiration autobiographique est certainement de défendre une certaine image idéale de soi, en mettant en avant la sensibilité et l'humanité du personnage principal. Or, la valeur et la complexité de Veneuse sont supposés transparaitre particulièrement dans ses rapports avec le sexe opposé, « le deuxième sexe » que sont encore les femmes à cette époque ${ }^{17}$. Cet article, au croisement de l'imagologie ${ }^{18}$ et des études génétiques, met en évidence et interprète la représentation des femmes dans Unhomme pareil aux autres à travers les amplifications, les suppressions et les réécritures dans les différentes versions publiées et manuscrites du texte. En reprenant l'hypothèse fort éclairante de Lourdes Rubiales qui propose d'appeler René Maran un "dandy ${ }^{19}$ ", nous proposons de le qualifier de « sentimental » au vu de l'importance des sentiments et des femmes dans sa vie et dans son roman d'inspiration autobiographique. Nous analysons ensuite la différence de traitement des personnages féminins blancs et noirs, en nous appuyant sur l'évolution $\mathrm{du}$ texte et le contexte historique de sa rédaction. Enfin, nous étudions le portrait changeant de la mal aimée Clarisse Demours, personnage encombrant pourrait-on dire, tant il menace l'image positive du héros Veneuse.

\section{Un homme pareil aux autres, le roman d'un dandy sentimental}

4 Si René Maran apparaît comme un dandy noir au temps des colonies ${ }^{20}$, c'est aussi un grand sentimental, ce que révèle son roman Un homme pareil aux autres. Jack Corzani avait d'ailleurs déploré son "lyrisme sentimental» et sa «pleurnicherie " ${ }^{21}$. Et pourtant, le sentimentalisme et la sensibilité sont la voie choisie par René Maran pour 
la valorisation de Veneuse, si proche de lui. Alors que l'«une des métaphores maîtresses de la colonisation [était] celle de la pénétration mâle de territoires vierges, de la domination virile de la gent indigène qui se trouv[ait] par la même féminisée " ainsi que le rappelle Roger Little ${ }^{22}$, Maran revendique pour son personnage l'ethos de l'intellectuel, sensible et amoureux, plutôt que l'ethos du conquérant brutal et viril. Le sentimentalisme est signe de distinction : il est le privilège et la malédiction des têtes bien faites et des cœurs généreux ${ }^{23}$. Et si, comme l'affirme l'ami de Jean Veneuse, Pierre Coulonges, "l'amour supprime toutes les barrières, même raciales. C'est lui le seul pacificateur, le seul colonisateur, le seul civilisé (HPA, 97) », le grand amoureux qu'est Veneuse est alors hautement civilisé. Certes, désigner l'amour comme le «seul colonisateur» est pour le moins paradoxal. Cette expression ajoutée à partir du manuscrit identifié comme le manuscrit $5^{24}$ oppose la colonisation telle qu'elle se pratique (guerre, pillage, exploitation) et telle que Maran l'a dénoncée dans Batouala, à l'amour vu comme la véritable source de la civilisation.

5 Le sentimentalisme est donc défense de l'humanité du noir et critique du colonialisme dans Un homme pareil aux autres. L'intrigue amoureuse y est effectivement compliquée par la question raciale. Résumons l'œuvre brièvement. À cause des préjugés sociaux qui pèsent sur les relations et les mariages mixtes, Jean Veneuse, épris d'Andrée, ne peut lui déclarer son amour et a décidé de partir pour l'Oubangui-Chari, colonie jugée très difficile ${ }^{25}$. Le départ est douloureux pour ce fonctionnaire colonial qui quitte la France, ses amis et sa bien-aimée à qui il n'a pas déclaré sa flamme. Sur le paquebot, il rencontre un vieil ami, Pierre Coulonges et la femme de celui-ci, à qui il confie son chagrin. Ceux-ci l'incitent à dévoiler ses sentiments à l'aimée, et le persuadent qu'il est aimé en retour. Mais cette première partie serait trop simple sans le personnage de Clarisse Demours. Celle-ci voyage pour rejoindre son mari, fonctionnaire colonial au Tchad. Elle s'éprend de Veneuse et ils entretiennent une liaison. À la fin de la première partie, Clarisse apprend la mort de son mari et souhaite suivre Veneuse mais ce dernier la repousse, n'ayant de sentiment que pour Andrée. La deuxième partie commence après cette rupture et l'installation de Veneuse à son poste, où, obsédé par Andrée, il ne vit que pour lire son courrier surtout depuis que les amants se sont enfin avoué leur amour. Veneuse prépare alors le « Retour en France » (sous-titre de l'extrait du Roman d'un nègre publié en 1924) ${ }^{26}$. Le dernier chapitre du roman, narré à la troisième personne, le montre arrivant au chevet de sa belle et lui demandant sa main. Elle accepte. Le livre s'achève sur la promesse du mariage, véritable "happy end " qui correspond à l'horizon d'attente du roman d'amour heureux.

6 Dans ce roman sentimental, la cristallisation amoureuse ${ }^{27}$ autour du personnage d'Andrée Marielle a une grande influence sur la description des autres femmes que le héros compare presque toujours à l'aimée. Veneuse ramène les sentiments éprouvés pour d'autres à ce qu'il ressent pour Andrée et les divers degrés d'amour décrits correspondent à une classification sociale et raciale des femmes, révélatrice du contexte historique du roman et des difficultés rencontrées par René Maran lui-même. Les femmes ont en effet été un sujet sensible et important dans sa vie. Ses lettres montrent qu'il a beaucoup souffert de n'être pas mariée 28 avant d'épouser Camille Berthelot à 40 ans. Cet homme de son temps s'inscrivait dans des cercles de sociabilité masculine (littéraire, de l'administration coloniale ${ }^{29}$ et des clubs sportifs) où les femmes étaient exclues et ne pouvaient être perçues comme des égales. Dans sa correspondance, il jugeait crûment de leur valeur en fonction de son idéal de «la 
Femme ", par exemple, quand il écrit à son jeune ami Charles Barailley en 1914 : «Les femelles d'ici [Fort Sibut], - ce ne sont pas des femmes, - me dégoûtent. Moins que dépotoirs, elles n'ont aucun sentiment profond. Je les exècre. Et moi qui aime tant les valeurs féminines, et la femme en général $!^{30}$ » Dans un précédent courrier envoyé à cet ami en 1912, où Maran annonce qu'il va se marier (ce qui n'aura pas lieu), il déclare :

$\mathrm{Ah}$ ! Ne va jamais aux colonies, ou ne vient qu'au Maroc, belle terre où les Françaises paraissent, toutes, belles. Je dis paraissent. Enlevé le corset, et la femme nue, on voit la déformation produite par les baleines et, aux pieds, par les talons hauts. Non seulement je suis misanthrope, mais encore, et surtout misogyne ${ }^{31}$.

Lourdes Rubiales a analysé cet aspect «misogyne » de la personnalité de Maran en l'interprétant comme une caractéristique du «dandy ${ }^{32}$, ce qui semble très pertinent car, si l'on en croit Baudelaire, dans Mon cœur mis à nu :

La femme est le contraire du Dandy. Donc elle doit faire horreur. La femme a faim, et elle veut manger ; soif, et elle veut boire. Elle est en rut, et elle veut être f...

Le beau mérite!

La femme est naturelle, c'est-à-dire abominable.

Aussi est-elle toujours vulgaire, c'est-à-dire le contraire du Dandy.

Bien sûr, ce ne sont pas là les mots de Maran. Mais ce dernier, comme beaucoup d'hommes de son temps, a été marqué par cette représentation patriarcale sexiste qui lie la femme à la nature, à l'instinct, au corps et à l'enfance. En témoigne cet autre extrait de lettre à Barailley datée du 6 décembre 1917 où Maran écrit : «Il ne faut pas en vouloir aux femmes. Elles ne sont pas plus méchantes que les hommes, ne serait-ce que parce qu'elles raisonnent moins leurs actes et sont esclaves de leurs nerfs et de leur sens... ${ }^{33}$ ». La femme de la première moitié $\mathrm{du} \mathrm{xx}^{\mathrm{e}}$ siècle reste piégée par deux stéréotypes : celui de la femme respectable, gardienne du foyer, et celui de la femme légère, instable, de petite vertu. Ce dualisme se retrouve chez Maran. D'une part il rêvait d'amour «pur» et, d'autre part, il était travaillé par le désir sexuel qui le poussait aux relations purement charnelles, à répétition ou sans lendemain. Ce dualisme puritain du corps et de l'esprit se doublait d'une dimension raciale du fait de l'idéologie raciste de la colonisation ${ }^{34}$. Ainsi, Bernard Mouralis explique que pour René Maran, la " peur du corps [...] semble bien pour lui être toujours du côté de l'Afrique " car toujours «[1]e charnel, le corporel, l'instinct [...] correspondent [...] à ce qui lui paraît caractériser le monde africain ${ }^{35} »$. Ce dualisme se retrouve dans le traitement des personnages féminins comme on le verra.

Un homme pareil aux autres pose finalement la question de l'«amour pur ", ou pour paraphraser Frantz Fanon, de l'" amour authentique $»^{36}$. Certes, dans Peau noire, masques blancs, publié cinq ans après le roman sentimental du soixantenaire René Maran, le jeune psychiatre révolté de 27 ans critiquait sévèrement ce récit. Il psychanalysait auteur et personnage, et concluait que " Jean Veneuse, alias René Maran n'est ni plus ni moins qu'un abandonnique noir ${ }^{37}$ ». Pour Fanon, Veneuse "alias René Maran» rationnalise sa névrose d'abandon par le problème racial et « cherche la tranquillité, la permission dans les yeux du Blanc ${ }^{38}$ ». Il serait un «inquiet collé à son corps » qui a «besoin d'une autorisation. Il faut qu'un Blanc lui dise : prends ma sœur ${ }^{39}$. » En dépit de ces critiques, comme l'a bien noté Mathieu Renault, Fanon et Maran ont plus en commun que ne veut bien l'admettre l'auteur de Peau noire, masques blancs ${ }^{40}$. Il revient à Maran le mérite d'avoir pointé l'importance de la réflexion sur l'amour et les «mariages fusionnaires ${ }^{41}$ » que Fanon aborde dans son essai. Maran critiquait le silence qui pesait sur ces questions et sur Un homme pareil aux autres, après sa parution. Ce 
silence était en réalité déjà patent dès la publication du Roman d'un nègre en 1924 puisque seul le chapeau faisait mention du mariage mixte, alors que l'extrait publié du roman n'avait rien à voir avec le sujet. Bien plus, l'extrait aurait pu commencer par les mots doux d'Andrée «Je vous aime. Je vous attends. Venez » qui précèdent le passage cité, mais ces mots sont effacés...

Figure 1 : Extrait paru dans la revue Je sais tout du 15 novembre 1924 de la première version d'Un Homme pareil aux autres, "Le roman d'un nègre " - illustration et titre

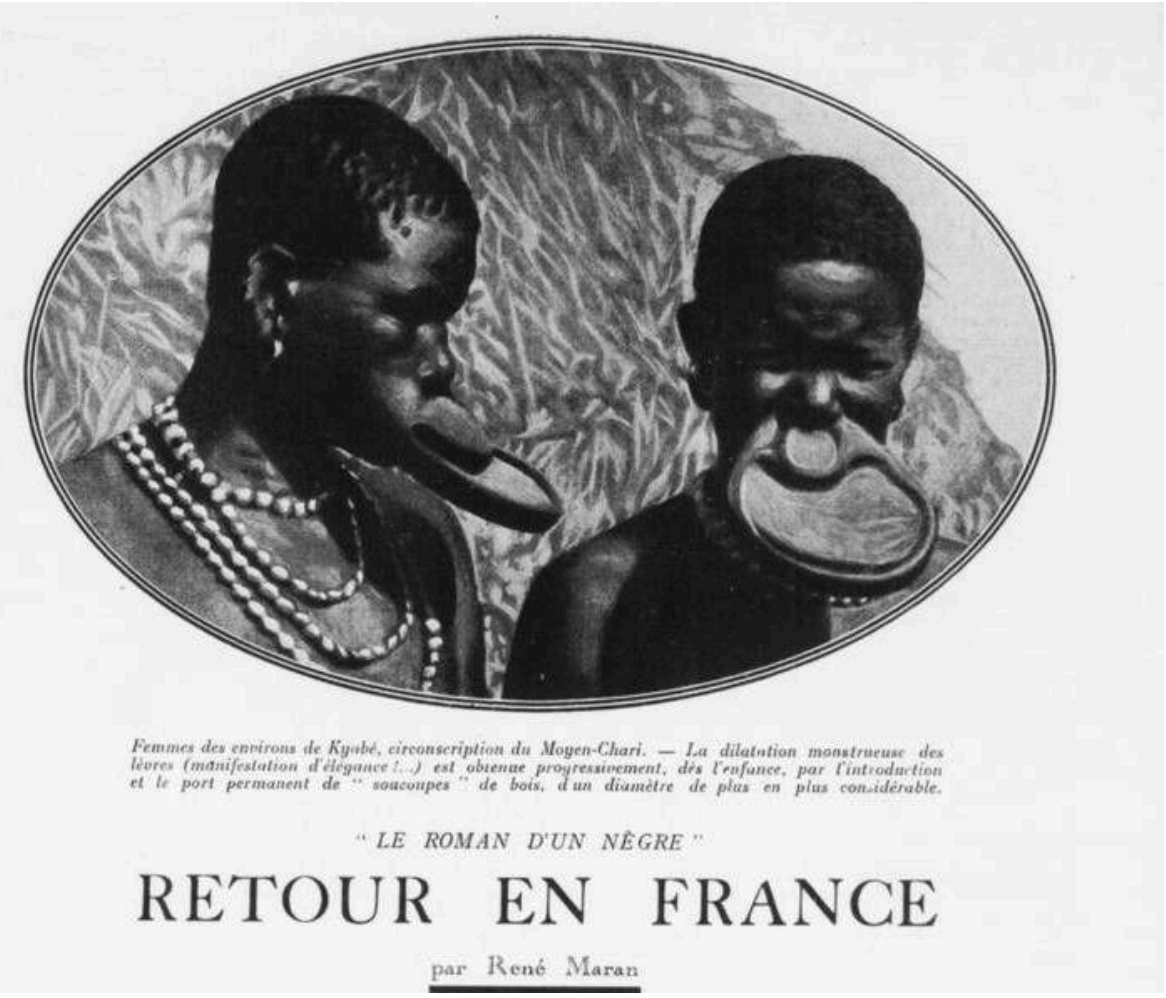

Figure 2 : Extrait paru dans la revue Je sais tout du 15 novembre 1924 de la première version d'Un Homme pareil aux autres, "Le roman d'un nègre " - chapeau et premiers mots de l'extrait.

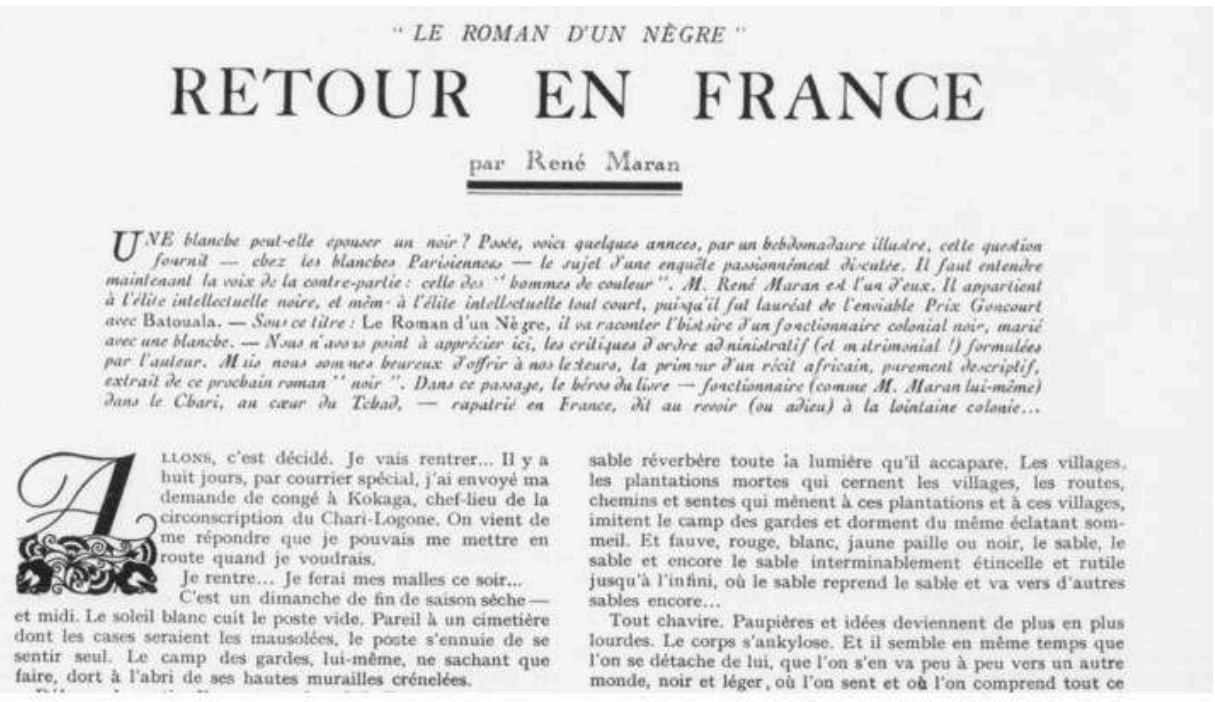


10 Contre ces silences, à l'instar des féministes qui rappellent que «le personnel est politique ", et à l'instar d'Annick Houël qui invite à lire les romans sentimentaux comme des « romans sociaux ${ }^{42}$ ", il est nécessaire de commenter de façon critique les représentations féminines d'Un homme pareil aux autres et de ses différentes versions.

\section{Femmes blanches et femmes noires dans Un homme pareil aux autres}

11 Grâce à la fiction d'une écriture de l'intime, quasi diariste, les femmes dans le roman sont décrites sans tabou. On peut les classer en deux catégories : les femmes blanches françaises, qui sont les plus nombreuses et importantes, et les femmes noires africaines, qui ne sont pas complètement ignorées.

\section{Les femmes blanches : un air de France et de l'aimée Andrée}

La première partie du roman évoque presque uniquement les femmes françaises blanches car c'est avec elles que sont tissés des liens d'amour et d'amitié. Elles renvoient, d'une façon ou d'une autre, à la figure d'Andrée. Simone dite Monna, amie d'un camarade qui accompagne Veneuse au port, est décrite dès le manuscrit 1 et dès Journal sans date (1927) comme "charmante", et "pleure». Veneuse l'embrasse: "Monna, en vous embrassant tout à l'heure c'est Andrée que j'embrassais, c'est à elle que j'adressais ma ferveur, ma tendresse, mes regrets» $(H P A, 19)$. Andrée est donc la deuxième femme évoquée. Après, paraît Clarisse Demours, d'abord ignorée par Veneuse qui est tout à son chagrin du départ. Avant que leur liaison ne commence, Veneuse l'associe déjà à Andrée: "Je sens que je souris à des visages, à une image: Monna, Mme Demours, Andrée. [...] Puis plus rien, le sommeil » (HPA, 26). L'évolution de cette phrase au fil des manuscrits ${ }^{43}$ montre que ce n'est que dans la version finale éditée du texte de 1947 que les trois « visages » se fondent en une seule "image », renforçant la vision onirique de ces femmes unies en une incarnation, et pour cause, puisque toutes incarnent la France, figure hautement féminisée, à qui Veneuse dit au revoir comme à une mère ou à une amante : «Ma France...! [...] Adieu à vous aussi, douce créature secrètement chérie !» (HPA, 24).

13 C'est également le lien à la France que symbolise Mme Coulonges, femme mariée, éduquée, qui est à la fois, un peu comme une sœur et une mère. Quand elle et son mari quittent Veneuse, Pierre Coulonges dit: «Tiens! Embrasse-la. C'est une bonne fille, prends un peu de France sur ses joues.» (HPA, 98). Mme Coulonges, quatrième personnage féminin du roman, est une figure importante. Veneuse explique qu'elle est " comme une grande sœur un peu maternelle" $(H P A, 82)$ et qu'elle a un "faux air d'Andrée » (HPA, 34). Bienveillante, elle réprimande néanmoins Veneuse au sujet de son aventure avec Clarisse. Mais face à ces reproches, Veneuse pense : « Le comble c'est que j'aime moralement Andrée dans Mme Coulonges et physiquement avec Clarisse. C'est insensé. Mais il en est ainsi » (HPA, 83).

Plus loin, il ajoute, dans un passage célèbre qui reprend le titre final (nous soulignons) du roman de 1947 :

Je veux qu'[Andrée] soit, avant d'être ma femme, mon amie. C'est pourquoi j'aime Mme Coulonges, qui d'ailleurs lui ressemble. C'est pourquoi je supporte sans

déplaisir Clarisse, qui a un peu de sa voix et de son port. Je ne sais pas, je ne sais 
plus et ne veux pas chercher à savoir quoi que ce soit. Ou plutôt, je ne sais plus qu'une chose : c'est que le nègre est un homme pareil aux autres, un homme comme les autres, et que son cœur qui ne paraît simple qu'aux ignorants, est aussi compliqué que peut l'être celui du plus compliqué des Européens.

J'aime Clarisse. J'aime Mme Coulonges. Et c'est André Marielle que j'aime. Elle seule, pas une autre. (HPA, 83)

Dans le manuscrit 1 , le passage est plus précis sur le mariage et les femmes (nous soulignons) :

Après tout, dans la plupart des cas, lorsqu'une femme se marie, elle devient la femme de son mari mais pas toujours son amie. Pourquoi le serait-elle? Depuis que le monde est monde, chez les noirs comme chez les blancs, l'homme qui obéit à ses instincts plus qu'à sa raison, n'a que très rarement cherché à comprendre la femme. Qu'un philosophe écrive qu'elle n'est qu'un petit animal usuel, on s'indigne tellement, par hypocrisie, on crie à la misogynie. Au fond, tel est cependant ce que l'homme pense vraiment de la femme... Et bien, moi, je veux qu'Andrée soit mon amie. C'est pourquoi j'aime madame Coulonges qui d'ailleurs lui ressemble. C'est pourquoi je supporte sans déplaisir madame Demours, qui a un peu de sa voix... Et puis, je ne sais pas, je ne sais plus et ne veux pas savoir quoi que ce soit... Le bonheur se fonde souvent sur l'hésitation ou le doute... Je ne vois bien qu'une chose : c'est que le nègre est un homme comme les autres. Si cela n'était, son cœur, qui ne parait simple qu'aux ignorants ou aux gens de parti-pris, ne serait pas aussi compliqué que celui du plus compliqué des européens.

J'aime madame Demours, j'aime madame Coulonges, et c'est Andrée Marielle que j'aime... Elle seule, pas une autre... $»^{44}$.

La comparaison de ces versions du texte montre que l'extrait est une célébration de l'amour pour Andrée et que la confusion des identités féminines n'est pas totale même si Veneuse voit en chaque femme blanche aimable, son aimée. Comme le révèle le premier manuscrit, Veneuse en revendiquant sa sentimentalité, s'oppose à la misogynie qui fait de la femme « un petit animal usuel». Son amour débordant n'est donc pas polyamour, mais se veut un hommage, aussi maladroit soit-il, aux femmes et à leurs différences, harmonieusement réunies en Andrée. L'image de gentleman de Veneuse est corroborée par l'étude des versions antérieures du texte, même si sa dénonciation de la misogynie a été finalement supprimée. On pourrait se demander pourquoi le passage a été retiré. Maran était-il conscient des failles de son personnage et a-t-il par conséquent évité d'en faire un moraliste décomplexé ? Il est évident que si la relation amicale de Veneuse avec Mme Coulonges est tout à son honneur, celle avec Clarisse compromet l'image de l'amoureux transi et fidèle.

17 La relation charnelle avec Clarisse s'oppose à la relation platonique avec Andrée et avec Madame Coulonges, amie maternelle. Cette opposition est confortée par l'introduction d'un autre personnage féminin blanc : Jacqueline, le premier amour de Veneuse, convoqué pour chasser l'image de Clarisse, la tentatrice. Arrivé au Congo, le héros est amer : «Congo, ô Congo! [...] Où est l'enthousiasme qui m'a jadis soulevé la première fois que je t'ai vu? Je t'exècre à présent. Je te hais.» (HPA, 109). Cette déclaration est peut-être à mettre en relation avec les incidents racistes subis par Maran au Congo en 1915 et $1916^{45}$ mais aucune allusion au racisme n'apparaît. Toujours est-il qu'à ce moment précis, Veneuse est très irrité par l'émerveillement de Clarisse face au paysage : «Elle frissonne contre moi. - Que c'est beau, que c'est beau ! Cela veut dire : 'Prends-moi. Je t'appartiens. Ce spectacle me transporte. Je le traduis en désir. Il faut que je me donne à toi pour exprimer ma joie'. » (HPA, 110). Veneuse résiste et pense " Je n'aime pas Clarisse. Je l'ai prise parce qu'elle ne m'eût point pardonné de ne pas le faire » (HPA, 111). Puis Veneuse songe à Jacqueline : «Elle m'aimait. Je faisais semblant de 
l'ignorer. Je l'aimais. » (HPA, 111). Jacqueline est donc un double d'Andrée qui reparaît en la mémoire de Veneuse pour lui « prouver qu'[il] n'oubliera pas plus Andrée Marielle qu'il ne [l'] a oubliée [elle Jacqueline] » (HPA, 113).

18 Clarisse la tentatrice est le personnage féminin blanc qui, présent dès les premières versions du texte, s'avère le plus complexe, avec un statut aussi ambivalent que celui des femmes africaines. C'est aussi ce que conclut Buata Malela dans son étude comparative de Journal sans date (1927) et d'Un Homme pareil aux autres (1947) ${ }^{46}$. Mais le critique note que Veneuse éprouve de l'attirance pour Clarisse et les femmes africaines, alors qu'en réalité, Veneuse refuse toujours de se présenter comme celui qui est attiré par les femmes : c'est elles qui le désirent et le tentent, ce qui est très différent. En outre, le récit oscille entre l'effacement, la reconnaissance et la défense des femmes noires.

\section{L'ambivalence vis-à-vis des femmes noires}

Absente de Journal sans date (1927), Adidja est l'unique personnage féminin noir décrit par Veneuse dans la deuxième partie de Défense d'aimer (1932) au chapitre 2, puis dans le manuscrit 4, le manuscrit 5 et dans la version de 1947 d'Un homme pareil aux autres. Veneuse la mentionne après avoir longuement décrit son chien et son amour pour cet animal :

Mon chien Piter et mes livres, voilà vraiment, ici, mes seuls amis. J'éprouve, toutefois, pour Adidja, ma petite compagne baguirmienne, qui rit pour le moment avec sa boyesse, [...] un sentiment assez bizarre, fait d'affection impondérable et de pitié profonde, que je ne parviens pas à analyser.

Mais bien que nous soyons tous deux de même race; bien qu'elle s'ingénie à me draper, à se draper de toute la riche lumière humaine dont déborde sa joie de vivre sans souci, elle est si loin de moi par sa formation sociale, ses goûts, son esprit, sa manière de voir, de comprendre, de sentir, et, devant les événements, de réagir ; elle est si effacée, si puérile et, pour tout dire, si discrètement résignée qu'elle ne compte guère à mes côtés que pour mémoire. $(H P A, 171)$

Le commentaire s'arrête là dans Défense d'aimer (1932), et donne lieu dans les versions postérieures (les manuscrits 4 et 5 , et la version de 1947) à une amplification selon une logique que nous avons déjà observée. Celle-ci ne se focalise pas sur Adidja mais sur les pensées de Veneuse :

N'ayant, elle et moi, rien de commun que notre couleur, nous formons, elle et moi, un de ces couples disparates comme il en existe tant par le monde, un de ces couples que, seule, l'œuvre de chair unit momentanément de temps à autre.

Je ne la traiterai pourtant jamais comme aurait trouvé sans doute naturel que je le fisse l'auteur de la circulaire officielle $n^{\circ} 60$, en date du 30 mai $1919^{47}$, que j'ai découverte l'autre jour en feuilletant les archives de ma subdivision.

Le ton de ce document me révolte, surtout le passage suivant: «Des incidents récents ", y lit-on, « ont démontré encore une fois l'influence fâcheuse que peuvent avoir les concubines indigènes de ceux des Européens qui ont la faiblesse de les considérer autrement que comme un instrument hygiénique. »

La formule est terrible et digne de Schopenhauer. L'Européen, le philosophe allemand lui servant d'interprète, y condense tout ce qu'il pense de la femme en général et de la sienne en particulier.

Donc, la femme, de quelque couleur qu'elle soit, n'est, à ses yeux, qu'un instrument hygiénique, rien qu'un instrument hygiénique. Un détail manque dans cet arrêt. La réciproque étant vraie, l'homme, de quelque couleur qu'il soit, n'est, lui aussi, pour la femme, qu'un instrument hygiénique.» 
21

Veneuse fait référence à une circulaire raciste sur «l'influence fâcheuse » des femmes
africaines traitées autrement que comme des «instruments hygiéniques » et affirme qu'il se refuse justement à traiter Adidja uniquement en simple «instrument hygiénique » mais la fin du passage opère un renversement. Alors qu'il était question des femmes colonisées, noires, en situation d'être dominées et méprisées par les lois coloniales, on aboutit à une conclusion surprenante, qui est comme une digression sur le malheur des hommes traités en "instruments hygiéniques» par les femmes... Ce renversement est d'autant plus osé que Clarisse, amoureuse de Veneuse et rejetée par lui, s'était précisément plainte de sa conduite en lui demandant : "Alors qu'ai-je été entre vos mains? Un jouet, hein! Rien qu'un jouet? Une sorte d'ustensile hygiénique en chair et en os [...] ?» (HPA, 195). Est-ce à dire que Veneuse prend sa revanche ici et suggère qu'il a été l'instrument hygiénique d'Adidja? Ou souhaite-t-il simplement nier l'idée d'iniquité et de domination subie par les femmes, en affirmant qu'il n'y a pas de déséquilibre entre hommes et femmes puisque ces dernières usent des hommes comme les hommes usent d'elles?

Ce renversement étonnant sur la question de la sexualité des femmes nous rappelle la lettre déjà citée envoyée par Maran à Manoël Gahisto en $1916^{48}$. Maran regrette de n'être pas encore marié, il est à Fort-Sibut au Congo et confie :

Je vous ai rarement parlé de mes rapports avec les femmes d'ici [...]. On n'a qu'à demander. Il n'y a jamais de refus. [...] Dieu sait que la fatuité ne m'aveugle pas. Il n'en est pas moins vrai que j'inspire hélas, de temps à autre des béguins...

L'autre soir, au moment où j'allais me coucher, vers onze heures, j'entendis frapper

à ma porte. J'ouvris. C'était une femme, vêtue comme toutes les femmes d'ici, de sa seule pudeur.

- Mo vé nié ? lui demandai-je en sango, ce qui signifie : Qu'est-ce que tu veux? Mbi ga tené mo balao (Je viens te dire bonsoir) - Tso oho ? (Cela seulement ?). Elle demeure confuse, la douce enfant. Entre nous, je répétai: Mo vé nié. Alors mon amoureuse se décide: Mbi vé lango na mo, qu'on peut traduire par « je t'aime » ou encore par « je te veux».

[...] Comme il y avait longtemps que je jeûnais, comme je sentais le désir faire le tour de mes reins [...] je consentis à l'accueillir momentanément dans ma couche, encore que son odeur forte affectât désagréablement mes narines délicates. Et puis elle est partie, non sans que je l'eusse rétribuée pour le service qu'elle m'avait rendu. Je ne la connais même pas. Et voilà comment il me faut vivre dans ces contrées. J'en rage. Un contact d'épiderme, rien de plus. On se prend et l'on se quitte. Que la vie est bête!

Cet épisode montre tout à la fois la satisfaction (consommation sexuelle) et l'insatisfaction de l'homme épris d'idéaux et de sentimentalité. Surtout, Maran passe sous silence le rapport d'inégalité socio-économique et donc le rapport de pouvoir qui existe entre lui et la femme africaine évoquée. Il semble considérer la démarche de la jeune femme comme un acte amoureux (un « béguin »), tout en reconnaissant qu'il lui a donné de l'argent. Il ne paraît pas comprendre que cette femme a donné son corps sans doute non par amour ou désir, mais pour gagner de l'argent et subsister.

Cette lettre montre aussi que ce sont les femmes qui vont chercher les hommes, et c'est effectivement ce qui est représenté dans la fiction. Clarisse infidèle à son mari est décrite en femme plus ou moins insistante, selon les versions et les différents manuscrits. Quand Veneuse pense «Je n'aime pas Clarisse. Je l'ai prise parce qu'elle ne m'eût point pardonné de ne pas le faire ", les versions antérieures du texte insistent sur l'absence prétendue de désir de Veneuse. Ainsi, on lit dans les manuscrits 1 à 3 (nous 
soulignons) : "Je n'aime pas Clarisse, c'est à peine si je la désire. " Puis, dans le manuscrit 4, nous trouvons : «Je n'aime pas Clarisse. Je l'ai prise parce qu'elle s'était donnée à moi [ajout manuscrit marginal] <ne m'eût point pardonné de ne pas le faire> ». La mention du désir impulsif attribué à Clarisse est raturée, mais l'idée demeure, avec celle du reproche de la femme qui refuse qu'on lui dise non... Dans Un homme pareil aux autres, Adidja, ou encore Touyalta, mère infanticide et femme infidèle, sont tout aussi coupables de céder à leurs pulsions sexuelles. Et faut-il rappeler qu'au cœur de Batouala se trouve l'infidèle Yassigui'ndja? Et que dire de la sensuelle fiancée de Djogoni ? Pour Marie-Hélène Koffi-Tessio, le portrait moral et physique de celle-ci est sexiste, et « révèle beaucoup de l'opinion de Maran sur les femmes noires et métissées : Mouhinda-Matiti séduit exclusivement par ses attraits physiques [...]. Plus tard elle est décrite comme superficielle, frivole, infantile, s'amusant de peu [...] et aimant surtout la toilette $[. . .]^{49}$.» Femi Ojo-Ade constate également: «La femme d'Afrique est facile, beaucoup trop facile selon Maran et Veneuse. Sa promiscuité s'exprime non seulement dans Batouala mais aussi dans Djogoni, Un homme pareil aux autres, etc..$^{50} »$

L'ambivalence de Maran vis-à-vis des Africaines ou, pour reprendre Veneuse au sujet d'Adidja, ce «sentiment assez bizarre, fait d'affection impondérable et de pitié profonde ", transparaît aussi dans la réécriture de deux passages de la première partie où est dénoncé le sort des femmes africaines colonisées. Le premier passage figure dans Journal sans date (1927) et Défense d'aimer (1932) et dans le manuscrit 1. Sur le bateau, Moynac et Faucheroux sont jaloux de la liaison de Veneuse avec Mme Demours et demandent aux dames si elles accepteraient de se marier avec un noir. Comme celles-ci répondent oui, Faucheroux décrète qu'il ne leur reste plus à qu'à " se marier avec des négresses ", ce à quoi Veneuse réplique : «Il ne faut pas médire des négresses » et ajoute : "Colons ou fonctionnaires, j'ai connu pas mal de blancs, en Afrique équatoriale, qui en avaient jusqu'à deux et trois comme concubines. » Cette phrase est réécrite à partir du manuscrit 4 pour devenir dans le texte de 1947 :

Il ne faut pas trop en médire, Faucheroux. Faites appel à vos souvenirs d'Afrique. En ce qui me concerne, colons, soldats ou fonctionnaires, j'ai connu, à une époque où les Européennes ne consentaient guère à s'expatrier, pas mal de blancs qui entretenaient de véritables harems. Croiriez-vous, par hasard, à la génération spontanée des mulâtres? Je ne vois pas, dans ces conditions, pourquoi, sous prétexte de défendre la race blanche, vous vous en prenez avec tant de jalousie aux gens de couleur, dont je suis.

Veneuse est moins assertif, et revient à sa condition d'homme noir civilisé ${ }^{51}$ suscitant la jalousie. Nous avons noté d'autres changements similaires dans certains passages où Veneuse évoque les méfaits de la colonisation.

Dans la version définitive du texte, le sort des femmes africaines est moins mis en avant, et le portrait de ces femmes est plus ambivalent du fait de l'introduction d'Adidja à partir du manuscrit 4. Pourtant dans le manuscrit 5, le plus fidèle à la version de 1947, apparaissent les histoires du peuple Sara et l'histoire de Touyalta, montrant l'intérêt ethnographique de Maran pour ces peuples. D'un côté donc, Veneuse le narrateur les décrit comme dotés d'une histoire et de cultures propres mais de l'autre, les personnages féminins africains sont peu valorisés et présentés comme inférieurs à l'idéal de la femme cultivée ${ }^{52}$ et vertueuse qu'incarnent Andrée Marielle et Mme Coulonges. En ce sens, nous ne rejoignons pas la lecture de Lourdes Rubiales qui déclare que « la femme africaine, incarnée par la compagne de Jean Veneuse, Adidja, de par son 
animalité positive, est loin d'incarner la "Bête noire" ou la femme du pantin. Elle deviendrait même, au fond, l'idéal de la femme, à condition d'être moins "inerte" dans ses rapports sexuels ${ }^{53}$." Lourdes Rubiales en évoquant "l'inertie» des femmes africaines, fait sans doute allusion à la lettre René Maran adressée à Manoël Gahisto le 7 juillet 1913 où il évoquait le souvenir d'un amour passé, parfait, car « partagé, à la fois intellectuel et sensuel ", à l'opposé des éphémères rencontres avec les femmes africaines. Maran confiait alors : "Je suis un délicat, un rêveur, un sentimental. Je ne pourrai donc jamais comprendre ni jamais aimer la femme indigène, inerte et simple réceptacle de spasmes désenchantés. Hélas! $!^{54}$ Jean Veneuse, dans son incompréhension et ses insatisfactions, semble bien être l'alter ego de l'écrivain, qui laisse transparaître son ambivalence pour les femmes noires africaines dans Un homme pareil aux autres, sans parvenir à analyser l'origine de ces sentiments formatés par le cadre historique et social de son temps.

Les portraits de femmes dans Un homme pareil aux autres sont donc de problématiques représentations de "la femme", européenne, et noire. Nous avons constaté que ces portraits ont été retouchés au fil des textes, notamment dans une logique d'amplification mais celle-ci peut servir paradoxalement à un recouvrement ou à un effacement des figures féminines blanches ou noires. Enfin, les personnages de femmes du roman ont aussi été retravaillés par le biais de suppressions et réécritures, ce que nous allons montrer à propos de Clarisse Demours.

\section{Le portrait problématique de Clarisse Demours}

Les corrections apportées concernant Clarisse n'ont pas pour simple objectif de complexifier le traitement de l'amour dans le récit et d'intensifier le périple sentimental du héros: elles servent à donner une meilleure image de Veneuse. La férocité du héros vis-à-vis d'elle est atténuée, leurs rapports sont relativement égalisés, et d'autres remaniements évitent de montrer Veneuse en amant infidèle et soumis à ses pulsions. Arrêtons-nous à quelques exemples.

\section{Le baiser}

Pour évoquer le premier baiser échangé avec Clarisse à la fin du chapitre de la première partie, dès le premier manuscrit, Veneuse recourt à un chiasme (nous soulignons) :
Mme Demours se serre contre moi. Je la sens nerveuse, palpitante, prête à se laisser prendre. Je la désire, mais ne l'aime pas. Elle ne m'aime pas, et me désire. L'odeur de son désir et de sa chair me trouble. Je n'en peux plus. Sa respiration est forte. Sa gorge se soulève à coups précipités. Elle pèse de tout son corps sur moi. Son pas s'attarde...

Le manuscrit 4 et la version de 1947 du roman ajoutent les phrases (en gras) suivantes :

Il ne faut pas que je me laisse tenter davantage. Je ne veux pas être que le cavalier servant de cette femme qui m'observe de biais. Elle a beau faire : je ne faiblirai pas. Je parviens à me dominer et à la conduire malgré [...]. Malgré le tumulte de mon sang en désordre, par les coursives désertes, j'ai néanmoins la force de la conduire jusqu'au seuil de sa cabine.

Les phrases ajoutées et soulignées montrent le sang-froid du "gentleman", avant le baiser, dont la scène a été dépeinte avec de légers changements au fil des versions du texte. Ainsi, des premiers manuscrits au Journal sans date (1927) et Défense d'aimer 
(1932) : « Brusquement, nos lèvres se joignent. Puis je la repousse, et me sauve comme un collégien. Ah! l'amour... » Puis, avec l'ajout proposé à partir du manuscrit 4 (en gras) et retenu dans la version de 1947, le désir brusque est remplacé par le brusque dégoût : « Nos lèvres se joignent. Brusquement, je la repousse, dégoûté de ma propre faiblesse, et me sauve comme si j'avais le feu à mes trousses. » Ce qui suit corrobore l'idée que la culpabilité masculine doit trouver un exutoire : "Andrée, que n'êtes-vous là ? Vous voyez ce à quoi j'ai failli me laisser entraîner ?» Finalement n'est-ce pas la faute à Andrée qui est absente si tout cela est arrivé ? Le passage se poursuit : «Ah ! l'amour... ». Cette interjection est moins ambiguë du fait de la mention d'Andrée, alors que dans les premières versions, on pouvait croire qu'elle renvoyait au baiser de clarisse...

\section{Vouvoiement ou tutoiement ? Rehausser le portrait de Veneuse}

Journal sans date montre le déséquilibre entre Veneuse et Clarisse : elle le vouvoie mais est tutoyée. Dans la version de 1947, les amants se tutoient, sauf quand Clarisse, repoussée par Veneuse, reprend le vouvoiement («Qu'ai-je donc été entre vos mains?» $H P A, 125)$. Le déséquilibre présent dans les premières versions du texte est donc effacé. Pourtant le passage au tutoiement chez Clarisse est dramatisé de façon intéressante dans Journal sans date (1927) car ce passage au «tu » se fait au moment où Clarisse déclare sa flamme à Jean Veneuse après l'annonce de la mort de son mari. «C'est vous que j'aime... C'est toi que j'aime, toi » devient dans Défense d'aimer (1932) et Un Homme pareil aux autres (1947) : " C'est toi que j'aime mon chéri... c'est toi que j'aime toi » (HPA, 123), ce qui rend Clarisse plus impudique, familière et sans retenue, alors que ce n'était pas le cas dans Journal sans date.

La valorisation du portrait de Veneuse a pour pendant la dévalorisation du personnage de Clarisse mais passe aussi par la suppression de certains commentaires désobligeants sur elle - de façon à préserver l'ethos digne du narrateur. Ainsi, dans la fameuse scène de l'arrivée au Congo, quand Clarisse s'émerveille de la beauté du pays, voici le récit de Veneuse du manuscrit 1 à Journal sans date chapitre $\mathrm{X}$ (cette fois, les suppressions qui surviennent entre Journal sans date [1927] et Défense d'aimer [1932] sont en gras) : «Je n'aime pas Clarisse. Je l'ai prise parce qu'elle s'est donnée à moi... Oh ! elle n'est pas plus mauvaise (ms 1)/ pire qu'une autre. Malheureusement pour elle, elle apparaît trop tard dans ma vie.». Le passage se poursuit, (les suppressions et modifications qui surviennent dans le manuscrit 4 sont notées en gras) : "-Ah ! que c'est beau, que c'est beau !... Je préfère me rappeler celle qui fut, autrefois, mon amie, il y a treize ans de cela. / Du même âge que moi, elle n'avait que la beauté de l'intelligence, souvent si adorable... / Son nom ? Jacqueline... »

Maran avait-il pressenti que le commentaire « elle n'est pas [plus mauvaise ms 1] pire qu'une autre, malheureusement pour elle » pourrait être lu comme une marque de prétention de la part de Veneuse ? Ces deux phrases disparaissent dans Défense d'aimer (1932) et dans Un Homme pareil aux autres (1947). En outre, dans la version finale du texte, le souvenir de Jacqueline est convoqué moins brutalement que dans le passage cité. La phrase "Je préfère me rappeler..." est supprimée et remplacée par : "Par quelle association d'idées ai-je passé pour en venir à celle qui fut mon adorable amie? / J'avais alors dix-huit ans. De cinq ou six ans plus âgée que moi, elle n'avait que la beauté de l'intelligence. Son nom ? Jacqueline. » Dans cette réécriture, le souvenir surgit sans 
que ce soit un choix, ce qu'exprimait «Je préfère ». Jean Veneuse paraît ainsi moins brutal vis-à-vis de Clarisse. De plus, on note que dans cette version, Jacqueline est plus âgée que Veneuse alors que dans les versions antérieures, les amis avaient le même âge. Veneuse passe ainsi pour l'homme gentil, sentimental, qui n'est pas au-dessus des femmes qu'il côtoie.

Enfin, dernier exemple parmi d'autres : lorsque Clarisse par dépit amoureux, commet l'erreur fatale qui consiste à injurier de façon raciste son amant en le traitant de "sale nègre ", la colère de Veneuse est palpable dans les premières versions du texte. Dans Journal sans date (1927) et Défense d'aimer (1932), il pense (ajout dans le texte final de 1947 mis en gras) : «Vous prétendiez m'aimer, Clarisse, et la première injure qui vous soit venue à la bouche est celle-là, la seule qu'une Européenne ne peut ni ne doit jamais adresser à un noir, [à partir du ms 4, "qu'elle prétend aimer ou qu'elle aime »] si elle l'aime vraiment... / Vous souffrez. Je le sais. À cause de cela, je vous pardonne. J'ignore de quelle manière un de vos congénères se serait [ms 4 se fût] conduit envers vous, s'il avait été [ms 5 eût été] dans ma situation. Mais je sais que vous m'aimez moins que vous ne le supposiez. [ms 4 Je sais, en revanche, que vous m'aimez moins que vous ne le croyez]. Ces deux mots-là et votre colère, viennent de m'éclairer sur votre "moi" secret. "

Les ajouts montrent que Veneuse insiste sur le tempérament instable qui serait au cœur de l'identité de Clarisse, ainsi décrédibilisée. Veneuse reste calme, réfléchi, mesuré, et c'est lui qui pardonne, qui comprend ou qui compatit comme le prouve cet autre ajout de la version de 1947, quand Veneuse doit annoncer à Clarisse la mort de son mari : «Dieu sait, Clarisse, que je m'étais promis de faire tout mon possible pour ne pas te faire de peine » $(H P A, 123)$.

\section{Conclusion}

Ces premières observations des modifications du texte invitent à considérer la complexité de l'écriture de l'amour et des femmes dans Un homme pareil aux autres. Il est clair que le dandy sentimental qu'était René Maran soignait l'image de son héros Jean Veneuse. L'ethos d'amoureux loyal et souffrant qui est mis en scène par l'écriture diariste n'était pas toujours compatible avec les événements racontés et il semble que l'un des objectifs de la réécriture ait été de renforcer l'image positive de ce héros, parfois au détriment de certains personnages féminins, et cela, sans que le dualisme corps/ esprit ne soit dépassé, au contraire... Nous pouvons comprendre l'effort pour soigner l'image de Veneuse comme une tentative de défense du héros noir dans un roman qui s'est fixé pour objectif de pourfendre le racisme avec les armes du sentimentalisme, mais c'est aussi une défense du double fictionnel et alter ego de Maran. Et ce ne sera pas là une véritable conclusion : en effet, l'approche génétique ouvre à l'in-fini du texte, en abordant la réflexion sur l'œuvre à travers les dynamiques de création et de réécriture... Dès lors, comment conclure ? est-il possible de conclure ? Certainement pas. Les chemins qu'ouvre la découverte de nouveaux documents (en particulier les manuscrits/tapuscrits, et les lettres) sont passionnants et si des chercheurs éminents ont déjà réfléchi à la genèse et aux processus d'écriture d'Un homme pareil aux autres, il reste un grand besoin de davantage de lectures et d'approches féministes de son œuvre monumentale. Nous espérons avoir apporté une modeste contribution à ce vaste chantier. 


\section{NOTES}

1. René MARAN, Un homme pareil aux autres, Paris, Éditions Arc-en-ciel, 1947. Nous utilisons cette édition comme référence indiquée comme HPA dans la suite du texte pour les citations, suivi du numéro de page.

2. Pour une mise au point provisoire sur l'histoire du texte et un état des lieux du dossier génétique de l'œuvre, voir dans le présent dossier notre article « Un Homme pareil aux autres au prisme des études génétiques. Histoire du texte et dossier génétique ».

3. Lourdes RUBIALES, «René Maran et l'écriture du moi », L'Autobiographie dans l'espace francophone, II : L'Afrique, Cadiz, Servicio de publicaciones de la Universidad de Cádiz, 2005, p. 53-83, p. 57.

4. L'œuvre maranienne « comporte quelque vingt-cinq volumes : quatre recueils de poèmes, deux romans autobiographiques, des nouvelles africaines et françaises, un roman/conte utopique (Le Petit Roi de Chimérie), les six romans de l'admirable cycle de la brousse africaine (dont Batouala n'a été que le premier, et qui inclut aussi plusieurs contes animaliers), de nombreux essais de nature historique ou ethnographique concernant divers pays africains, et une série de biographies consacrées à de grandes figures comme Livingstone, Savorgnan de Brazza, Félix Éboué, Bertrand Du Guesclin, et à treize «Pionniers » de l'Empire français en Amérique et en Afrique (série publiée en trois volumes et incluant des études sur d'illustres explorateurs comme Jean de Béthencourt, Jacques Cartier, Nicolas de Villegaignon, Samuel Champlain, Pierre Belain d'Esnambuc et Cavelier de la Salle, notamment) » comme le récapitule l'argumentaire de l'appel à communication du colloque international «René Maran, la France, l'Afrique et la Littérature » prévu le 25 et 26 novembre 2021 et organisé par l'UCAD de Dakar et l'Université des Antilles en partenariat avec l'Université de Guyane, l'ITEM-CNRS et l'Université Paris 8.

5. Voir la lettre du 23 février 1921 citée par Charles KUNSTLER, «Le cœur, l'esprit et la raison », Hommage à René Maran, Présence Africaine, 1965, p. 51 : « Tout au long de la traversée j’ai pris des notes, ici et là, rectifié certaines de mes observations antérieures. Les unes et les autres participeront au Roman d'un nègre. [...] Il m'aidera à revivre un passé qui m'est cher, parce que je l'avais peuplé de belles illusions. "

6. Titre de l'un de ses recueils de poésie (René MARAN, Le Livre du souvenir, Paris, Présence africaine, 1958).

7. La première version intégrale publiée du texte en 1927 s'intitule ainsi éloquemment Journal sans date.

8. Voir les travaux de l'historienne Anne-Marie SOHN sur l'amour, la sexualité et la correspondance au $\mathrm{xx}^{\mathrm{e}}$ siècle, et son entretien accordé à L'Express le 8 août 2002, "Les Années folles par Anne-Marie Sohn ", URL : https://www.lexpress.fr/culture/livre/7-les-annees-follespar-anne-marie-sohn_818091.html, consulté le 23 juillet 2021.

9. Roger LITTLE, «Blanches et noirs aux années 20 », Regards sur les littératures coloniales - Afrique francophones: Approfondissements - Tome II, Jean-François Durand (éd.), Paris, L'Harmattan, 1999, p. 7-50, p. 9-10.

10. Dans les premiers manuscrits d'Unhomme pareil aux autres, Jean Veneuse raconte à Mme Demours des histoires coloniales de ce type.

11. Roger LITTLE, «Blanches et noirs aux années 20 », art. cit., p. 40.

12. Il est à noter que le dernier chapitre du roman est néanmoins écrit à la troisième personne, et que l'alternance entre première personne et troisième personne est prégnante dans tout le roman et ce, dès le premier chapitre. Cette énallage nécessiterait une étude stylistique approfondie. 
13. Buata MALELA, « René Maran et la "question noire" en France : Stratégies et prises de position dans le champ intellectuel des années vingt et trente ", Paris, Présence africaine, 2013/1, $\mathrm{n}^{\circ}$ 187-188, p. 121-138, p. 135.

14. Tina Harpin a consacré une étude littéraire féministe du double thème de l'amour et des femmes dans la littérature guyanaise (Tina HARPIN, «Amour, colère et nausée: portraits de femmes guyanaises, de Damas à Thébia ", dossier « "Mines de rien" : L'Antillaise et l'Afropéenne face aux tropologies, entre mythes et réalités au fil du temps ", Kathleen Gyssels et Jacqueline Couti (coord.), Essays in French Literature and Culture, n 56, octobre 2019, p. 93-108). Cet article à quatre mains prolonge ces réflexions, en se concentrant sur René Maran et en intégrant les apports de l'étude génétique.

15. Lourdes RUBIALES, « René Maran et l'écriture du moi », art. cit., p. 57.

16. Voir la lettre de Maran à Frédéric Jacques Temple du 5 avril 1948 où il écrit à propos d'Un Homme pareil aux autres (nous soulignons) : «Certes, mon roman n'est pas un chef-d'œuvre ! Nul ne le sait mieux que moi. Mais il est l'œuvre d'un Français de couleur de bonne volonté, et qui, malgré tout ce qu'on a pu dire ou écrire de lui, n'a jamais versé dans l'européanophobie. À preuve ce que je dis de mes congénères dans tous mes ouvrages, même dans Un Homme Pareil aux Autres. Au fond, peu importe. L'essentiel est de faire, seul parfois contre tous, son devoir social. Travailler de son mieux, à son rang, à sa place, selon son intelligence, telle est la règle du jeu. » ( Six lettres inédites de René Maran à Frédéric Jacques Temple - Autour d'Un homme pareil aux autres », Présence Africaine, 2013, 1-2, n 187-188, p. 175-182).

17. Rappelons que l'essai historique de Simone de Beauvoir sur la condition féminine est publié deux ans après la publication d'Un homme pareil aux autres. Simone de BEAUVoIR, Le Deuxième Sexe, t. 1, Les faits et les mythes, t. 2, L'expérience vécue, Paris, Gallimard, 1949.

18. Yves Chevrel explique que «le terme imagologie s'est imposé à la fin des années 1960, à la suite des travaux du comparatiste belge Hugo Dyserinck, pour caractériser une branche particulière des études comparatistes, celle qui concerne l'imagerie culturelle, en particulier les représentations de l'étranger qui sous-tendent les œuvres littéraires » et note que « l'imagologie interroge l'altérité sous toutes ses formes». Yves CHEVREL, «Réception, imagologie, mythocritique: problématique croisées", L'Esprit créateur, 2009, vol.49, n 1 , Les Études de réception en France / Reception Studies in France, 2009, p. 9-22, p. 10.

19. Lourdes RUBIALES, "Un dandy noir entre Bordeaux et l'Oubangui-Chari (1909-1921) ", Les Lettres romanes, $\mathrm{n}^{\circ}$ 70, $\mathrm{n}^{\circ}$ 1-2, 2016, p. 159-181.

20. Ibid.

21. Jack CORZANI, «La Littérature des Antilles-Guyane françaises », Fort-de-France, Désormeaux, 1978, p. 205, cité par Lourdes RUBIALES, « René Maran et l'écriture du moi », art. cit., p. 80.

22. Roger LITTLE, « Blanches et noirs aux années 20 », art. cit, p. 8-9.

23. Dans le chapitre IV de la deuxième partie, Jean Veneuse fait ainsi le portrait d'André Suarès qu'il admire : « Définir André Suarès n'est pas besogne facile. Il se veut viril, et parvient à l'être. Il est cependant plus féminin que viril et moins féminin que femmelin pour reprendre un mot des Goncourt, que les Goncourt ont d'ailleurs emprunté au vocabulaire du moyen âge. On ne trouve pas en effet dans ses écrits cette force équilibrée, cette santé physique et intellectuelle que les Latins mettaient dans le mot vir. Son humeur, qui se ressent toujours de l'heure et du moment, varie comme plume au vent. Un rien le froisse. Un rien l'irrite. » $(H P A, 198)$.

24. Pour une description des manuscrits du dossier génétique d'Un homme pareil aux autres, voir notre article déjà cité «Un homme pareil aux autres au prisme des études génétiques. Histoire du texte et dossier génétique ». Manuscrit 1 et $4:$ « L'amour supprime toutes les barrières... C'est lui le seul pacificateur, le seul civilisé... ». Manuscrit $5:$ : l'amour supprime toutes les barrières. C'est lui le seul pacificateur, le seul colonisateur, le seul civilisé... ». 
25. René Maran la qualifiait de « colonie-dépotoir où l'on déverse, vaste champ d'épandage, les fonctionnaires ou agents dont la conduite laisse à désirer ou dont la Métropole désire entendre parler le moins possible ». René MARAN, Félix Eboué, grand commis et loyal serviteur 1885-1944, [1957], Paris, L'Harmattan, coll. « Autrement mêmes », 2007, p. 19.

26. René MARAN, Le Roman d'un nègre, extrait, revue Je sais tout, 15 novembre 1924.

27. Cette notion est proposée par Stendhal dans De l'amour (1822). Stendhal s'inspire du phénomène biologique de cristallisation d'un rameau d'arbre plongé dans une mine de sel pour désigner par métaphore, la construction imaginaire qui entoure la figure de l'aimé(e) : «Ce que j'appelle cristallisation, c'est l'opération de l'esprit, qui tire de tout ce qui se présente la découverte que l'objet aimé a de nouvelles perfections. » La cristallisation amoureuse marque le début de l'expérience amoureuse et se trouve stimulée par la séparation d'avec l'aimé(e) et les doutes, ce qui correspond parfaitement à la situation de Veneuse, animé d'un amour neuf, puissant (avant la routine et les désillusions que peut provoquer le mariage) et habité par une rêverie qui se nourrit de l'absence d'Andrée.

28. René MARAN, lettre du 14 août 1916 à Manoël Gahisto : «Vraiment il y a longtemps que j'aurais dû me marier. » Manoël GAHISTO, La Genèse de Batouala, Ms 24, inédit, s.d., p. 73, fonds René Maran de la bibliothèque numérique de l'Université Cheikh Anta Diop de Dakar [consulté le 23 avril 2021] : http://bibnum.ucad.sn/viewer.php?c=rares\&d=1\%5fms\%5f24.

29. Dans sa lettre à son ami Paul Culine du 13 janvier 1910, Maran écrit ainsi : « Nous causons de femmes toute la journée car avec les amis que j'ai laissés un peu partout à Bordeaux, ce sont les seuls regrets de France que j'ai », Manoël GAHISTO, La Genèse de Batouala, op. cit., p. 7.

30. René MARAN, lettre à Charles Barailley du 18 mai 1914 numérisée par la plateforme Manioc, des SCD de l'université des Antilles et de l'université de Guyane.

31. René MARAN, lettre à Charles Barailley du 11 octobre 1912 numérisée par la plateforme Manioc, des SCD de l'université des Antilles et de l'université de Guyane.

32. Lourdes RUBIALES, « Un dandy noir », art. cit.

33. René MARAN, lettre à Charles Barailley du 6 décembre 1917 numérisée par la plateforme Manioc, des SCD de l'université des Antilles et de l'université de Guyane.

34. Maran, formé à l'école française et coloniale, avait intégré cette vision du monde, même s'il l'a beaucoup interrogée. Il écrit par exemple à Charles Barailley: "La civilisation rend malheureux ceux qu'elle subjugue. Elle ne détruit pas en eux, en les pénétrant, les coutumes ataviques. » Lettre à Barailley, Grimari, 3-3-1914, FPBB, cité Lourdes RUBIALES, « Un dandy noir », art. cit., p. 175.

35. Bernard MOURALIS, « René Maran, un écrivain mauriacien?» Hommage à Bernard Alluin, Lille, PUL, 2005, p. 92, cité par Lourdes RUBIALES, « René Maran et l'écriture du moi », art. cit., p. 76.

36. Frantz FANON, Peau noire, masques blancs, Paris, Seuil, 1952, p. 33.

37. Ibid., p. 64.

38. Ibid., p. 61.

39. Ibid., p. 53-55.

40. Mathieu Renault remarque qu' « [i]l y a en effet chez Maran des formules qui auraient pu tout aussi bien être celles de Fanon » puis se demande : « Doit-on se limiter à un tel constat d'analogie entre les discours de Fanon et de Maran? Ces ressemblances, ces correspondances, ne révèlentelles pas plutôt un certain héritage, une dette du psychiatre martiniquais à l'égard de son aîné, dette inavouée et à bien des égards inavouable dans la mesure où son effort est précisément de s'arracher, de se définir en contrepoint de tout ce que Maran-Veneuse représente. Mathieu RENAULT, «Amour de la race ou amour au-delà des races ? Frantz Fanon, lecteur de René Maran ", Présence Africaine, 2013/1, n 187-188, p. 231-244, p. 232 et 240. 
41. Maran reprend fréquemment cette expression, inspirée par Victor Schoelcher pour désigner les mariages mixtes. Voir par exemple la note de lecture "Maurice Bedel et les mariages fusionnaires » conservée sous forme de tapuscrit à l'UCAD.

42. Annick Houel, Le Roman d'amour et sa Lectrice: une si longue passion. L'exemple Harlequin, Paris, Montréal, L'Harmattan, 1997.

43. Ms 1 : Je sens que je souris à un visage, à des visages...Monna, madame Demours...Andrée.

Ms 3 : Je sens que je souris à un visage, à des visages... Monna, madame < la femme de Demours>, Andrée.

Ms 4 : Je sens que je souris à un visage, à des visages [ajout à la marge] <à un visage> : Monna, Mme Demours, Andrée.

44. Dans les manuscrits suivants, le texte reste inchangé à part le nom de Mme Demours qui devient Clarisse (sur ce point voir infra), et c'est à partir du manuscrit 4 que l'extrait prend sa forme définitive.

45. Sur ce point voir Lourdes RUBIALES, «Désillusion et frustration. L'administration coloniale contre René Maran ", art. cit, p. 221-222.

46. Buata Malela note que l'attitude de Veneuse vis-à-vis « de Clarisse Demours ne diffère pas de celle qu'il pourrait adopter avec une femme africaine ». Buata MALELA, « Authenticité et réécriture de soi dans Journal sans date / Un homme pareil aux autres de René Maran ", René Maran: une conscience intranquille, Roger Little (éd.), Lecce, Interculturel Francophonie, 2018, p. 159-182, p. 171.

47. Cette circulaire est aussi citée et critiquée dans une note de lecture sur Alan Paton et Christine Garnier, conservée sous forme de tapuscrit à l'UCAD.

48. René MARAN, Lettre du 14 août 1916 à Manoël GAHISTO, La Genèse de Batouala, op. cit.

49. Marie-Hélène KoffI-TESSIO, «Djogoni, le roman d'un Métis ou l'inanité de la mission civilisatrice », dossier « René Maran », Lourdes Rubiales (dir.), Francofonía, n 14, 2005, p. 39-62 ; p. 58.

50. Femi OJO-ADE, René Maran, Paris, Nathan, p. 16.

51. Maran n'approuvait pas tous les mariages interraciaux et déconseillait par exemple «tout mariage entre une Européenne, quel que soit le milieu social dont elle est issue, et un de ces trop nombreux indigènes de l'Afrique noire [...], les pagnes ou les boubous leur servant de vêture, et les cases en torchis leur servant d'habitation. » René MARAN, « Le point de vue de René Maran sur les mariages coloniaux", manuscrit de la Bibliothèque de l'UCAD, http://bibnum.ucad.sn/ viewer.php?c=rares\&d=023\%5fReneMaranMariagesColoniaux [consulté le 22 août 2021].

52. Cet aspect est souligné dans le manuscrit 1 , où Jean Veneuse est impressionné par la culture de Mme Coulonges qui « sait du grec » et connaît la pièce d'Ibsen, Un ennemi du peuple.

53. Lourdes RUBIALES, « René Maran et l'écriture du moi », art. cit. p. 77.

54. Manoël GAHISTO, «La genèse de Batouala ", Hommage à René Maran, Présence Africaine, 1965, p. 125. Cette lettre est aussi citée par Marie-Hélène KOFFI-TESSIO, art. cit., p. 58.

\section{RÉSUMÉS}

Un homme pareil aux autres est un roman d'inspiration autobiographique de René Maran, réécrit des années 1920 jusqu'à sa version définitive de 1947. C'est en réalité un roman d'amour dont le sentimentalisme a été peu commenté, ou carrément déconsidéré. Cet article analyse les enjeux de 
l'écriture du sentiment en étudiant le rapport de Jean Veneuse aux femmes, et l'évolution du portrait de celles-ci dans les différentes versions du texte. Un homme pareil aux autres est le roman d'un dandy sentimental où s'expriment certains préjugés sexistes quand d'autres préjugés racistes sont combattus. Veneuse ne parvient pas à exprimer la condition des femmes noires pas plus que celle des femmes blanches, toutes vues à la lumière de l'image idéalisée de l'aimée Andrée Marielle. Dans l'ombre, Clarisse, au statut aussi ambivalent que celui des femmes africaines, est le témoin, dans les réécritures du récit, de la position difficilement défendable parfois, du héros présenté en amoureux transi.

Un homme pareil aux autres is a novel inspired by the real life of René Maran, rewritten from the 1920s until its final version in 1947. In fact, it is a romance novel whose sentimentality has received little attention or was squarely belittled. This article analyses what is at stake in this sentimental writing by studying Jean Veneuse's relationship to women and the evolution of their portrayal in the different versions of the text. Un homme pareil aux autres is the novel of a sentimental dandy in which certain sexist prejudices are expressed whereas, on the other hand, certain racist prejudices are fought against. Veneuse fails to express the condition of black women or white women, all seen in the light of the idealized image of the beloved Andrée Marielle. Another white woman, Clarisse, remains in the shadows as she witnesses the indefensible behavior of the narrator who presents himself as a bashful lover. Her status is as ambivalent as the one of the African women characters and remains awkward in the various versions of the novel.

\section{INDEX}

Mots-clés : René Maran, femmes, racisme, études génétiques, manuscrits, roman d'amour, inspiration autobiographique, histoire coloniale

Keywords : René Maran, women, racism, genetic criticism, manuscripts, love novel, autobiographical inspiration, colonial history

\section{AUTEURS}

\section{TINA HARPIN}

Université de Guyane

\section{LAURA GAUTHIER BLASI}

Universidad Europea de Madrid 